\title{
Degree of Rate Control Approach to Computational Catalyst Screening
}

Christopher A. Wolcott ${ }^{\mathrm{a}, \$}$, Andrew J. Medford ${ }^{\mathrm{b}, \mathrm{s} \$}$, Felix Studt ${ }^{\mathrm{b}, \mathrm{c}}$, and Charles T. Campbell ${ }^{\mathrm{a}, \mathrm{d}, *}$

${ }^{a}$ University of Washington, Department of Chemical Engineering, Seattle, WA 98195, USA

${ }^{b}$ Department of Chemical Engineering, Stanford University, Stanford, CA 94305, USA

${ }^{c}$ SUNCAT Center for Interface Science and Catalysis, SLAC National Accelerator Laboratory, 2575 Sand Hill Road, Menlo Park, CA 94025, USA

${ }^{\mathrm{d}}$ University of Washington, Department of Chemistry, Seattle, WA 98195-1700, USA

${ }^{\$}$ These authors contributed equally.

*corresponding author, campbell@ @ chem.washington.edu

\begin{abstract}
A new method for computational catalyst screening that is based on the concept of the degree of rate control (DRC) is introduced. It starts by developing a full mechanism and microkinetic model at the conditions of interest for a reference catalyst (ideally, the best known material) and then determines the degrees of rate control of the species in the mechanism (i.e., all adsorbed intermediates and transition states). It then uses the energies of the few species with the highest DRCs for this reference catalyst as descriptors to estimate the rates on related materials and predict which are most active. The predictions of this method regarding the relative rates of twelve late transition metals for methane steam reforming, using the $\mathrm{Rh}(211)$ surface as the reference catalyst, are compared to the most commonly-used approach for computation catalyst screening, the Nørskov-Bligaard (NB) method which uses linear scaling relationships to estimate the energies of all adsorbed intermediates and transition states. It is slightly more accurate than the NB approach when the metals are similar to the reference metal $(<0.5 \mathrm{eV}$ different on a plot where the axes are the bond energies to $\mathrm{C}$ and $\mathrm{O}$ adatoms), but worse when too different from the reference. It is computationally faster than the NB method when screening a moderate number of materials $(<100)$, thus adding a valuable complement to the NB approach.
\end{abstract}


It can be implemented without a microkinetic model if the degrees of rate control are already known approximately, e.g., from experiments.

KEYWORDS: Catalyst screening, computational catalysis, density functional theory, degree of rate control.

\section{Introduction}

The ubiquity of heterogeneous catalysis in the production and utilization of fuels, bulk chemical processing and pollution clean-up means that even minor improvements in catalyst performance could result in massive improvements in the utilization of natural resources, environmental benefits and process performance[1-3].Thus, the motivation to find better catalyst materials is great.

One method for finding better catalysts has been high-throughput experimental screening of many materials for their catalytic performance (activity, selectivity, lifetime)[4]. However, this is costly, and with the evolution of fast computational methods that can predict catalytic reaction rates for different materials with increasing speed and accuracy[5], one expects that computational pre-screening of materials for catalytic activity or selectivity to find the best candidates for experimental testing will soon become a faster route to the discovery of new catalysts. Indeed, there have already been some successes in using high-throughput computational screening to accelerate the discovery of new catalysts[6-12]. We describe here a new approach for computational screening of new catalysts that relies on the degree of rate control (DRC)[13] to identify descriptors and estimate reaction rates on new materials. The new approach does not rely on a kinetic model (provided the degrees of rate control are known) and, in some circumstances, is faster and more accurate than the most widely used method.

Perhaps the most successful method for computational screening of solid catalysts has been the descriptor-based, linear-scaling approach developed by Nørskov, Bligaard and coworkers[5,6,14-17], which we will refer to here as the "NB method". It relies on the following approach to calculate the rate or selectivity on a new material: 
1. Determine the reaction's mechanism and active site on a good known catalyst in a certain class of materials through a combination of experiment and theory. We will refer to this as the reference material (RM).

2. Develop a microkinetic model for that mechanism that can reproduce experimental trends of the reaction rate for the RM at a variety of conditions, but relies on knowing the energies of all the adsorbed intermediates and transition states involved in every elementary step as it occurs on that RM.

3. Assume that the same mechanism and active site of the RM apply to all materials in its class, then use electronic structure theory to compute the energetics of all necessary adsorbed intermediates and transition-states for a representative set of materials from the same class as the RM.

4. Develop "linear scaling relations" which relate all the needed intermediate/transitionstate energies to one or two key "descriptors" (typically the adsorption energies of atomic species such as $\mathrm{C}^{*}, \mathrm{O}^{*}, \mathrm{~N}^{*}$, etc.). The necessary intermediate/transition-state energies of any given new material can then be estimated by knowing only these few descriptor energies, which can be computed using an electronic structure method such as density functional theory (DFT). This step relies on the demonstrations by Nørskov's group that the energies of most adsorbed intermediates vary approximately as linear combinations of the adsorption energies of two elemental adatoms (e.g., $C^{*}, \mathrm{O}^{*}$ or $\left.\mathrm{N}^{*}\right)[14,18,19]$, and that the activation energy for an elementary surface reaction varies linearly from material to material with the changes in the reaction's internal energy (i.e., a form of Brønsted or Evans-Polyani relationship).[15,20]

5. Choose a new material and calculate the "descriptor" energies (e.g., the energies of two adsorbed adatoms, $\mathrm{C}^{*}$ and $\mathrm{O}^{*}$ ). Use the "scaling" relations from step 4 to estimate the energies of all the intermediates and transition states in the mechanism, and from these energies, calculate the rate for the new material to the product(s) of interest at the desired reaction conditions. Since a typical microkinetic model requires the energies of more than 10 adsorbed intermediates and 10 transition states, and these are estimated using only one or two descriptors that are much faster to calculate than even one transition state, this can reduce the computational time needed to calculate rates on a new material by orders of magnitude, provided the linear scaling relations have already been developed. 
6. Repeat step 5 for all the new materials to be screened, and generate a volcano plot of rates versus descriptors' energies.

This approach results in a plot of catalytic activity as a function of the one or two descriptor variables which exhibits a characteristic "volcano" shape that determines the optimal descriptor values for maximizing the rate, within a given reaction mechanism and active site structure. We denote this type of plot as a "NB rate volcano".

Here, we introduce an alternate approach to the method outlined above which focuses instead only on the most relevant energies that most directly control the catalyst's activity. This can significantly simplify the analysis of reactions in any multi-stepped catalytic reaction mechanism, like methane reforming, since a small change in the internal energy of any particular intermediate or transition-state energy usually does not have any significant effect on the reaction rate, which is instead sensitive to only a few of these species' energies[13]. It is therefore reasonable to assume that one can simplify computational screening by identifying a small number of rate-controlling species (intermediates and transition states) whose energies most strongly affect the reaction rate, and develop a screening model based on these energies alone.

The "degree of rate control" (DRC) developed by Campbell et al[13] is a rigorous method for quantifying the extent to which each species' standard-state free energy affects the net reaction rate to any product (or the rate of consumption of any reactant). The DRC of any such species $i$ (which could be any intermediate or any transition state) is defined as:

$$
X_{i}=\left(\frac{\partial(\ln r)}{\partial\left(\frac{-G_{i}}{R T}\right)}\right)_{G_{j \neq i}},
$$

where $r$ is the rate to the product of interest at the chosen reaction conditions, and $G_{j}$ is the standard-state Gibbs free energy of species $j$. The DRCs, $X_{i}$, will depend on reaction conditions and the reaction mechanism investigated. There is a useful analogy between the DRC and the concept of a rate-limiting step: in the case that there is a single mechanism and a single ratelimiting step, the DRC of that transition-state will be unity, while the DRC for all other transition-states will be zero[21]. For more complex reactions without a clearly defined ratelimiting step, multiple transition-states will have a non-zero DRC. Furthermore, the $X_{i}$ of all transition-states will sum to one for each serial pathway through a reaction network, while the sum over $X_{i}$ of all adsorbed intermediates will fall between zero and a small negative integer[22- 
24]. For most reactions only a few transition-states and adsorbed intermediates exhibit nonnegligible DRC,[25,26] with all other transition-states and intermediates being essentially zero.

By quantifying the impact that changing the standard-state free energy of a particular species in a reaction landscape will have on the net reaction rate, it is straightforward to see that any species whose degree of rate control has a large magnitude can be categorized as a "ratecontrolling" species. By shifting the focus from the kinetics of a large number of reaction steps to the standard-state free energy of a small number of chemical species, it is substantially more straightforward to determine descriptors that are useful in finding improved catalysts. This is especially true since the entropy contribution to the standard-state free energy of each adsorbed species differs very little across different materials[27,28], whereas the internal energy dominates the differences in standard-state free energies between different materials. Thus, instead of looking for a catalyst with a faster rate directly, all that is necessary is to identify a catalyst with improved zero-Kelvin adsorption/transition-state internal energies for a small number of species. For example, when there is a single rate-determining transition state, one wants a material with a lower internal energy for that transition-state. On the other hand, when there is a single rate-determining intermediate adsorbing so strongly that it blocks the active sites, one wants to raise its internal energy. Often these quantities are correlated, so that a lowerenergy transition state will also often lead to a more strongly adsorbed intermediate. It is therefore necessary to include all species with significant DRCs. The best catalyst might be, for example, a material where this undesirable correlation is weakest.

The NB method is a very powerful approach for computational catalyst discovery, since it offers a fast way to estimate catalyst activity. However, it has some shortcomings. Most obviously, there exists scatter about each of the linear scaling relationships due to real errors in assuming that each energy scales linearly with the descriptor(s), leading to inaccuracies in their energy estimates and thus in the rate predictions. Additionally because of the large number of species-surface combinations one needs to calculate by quantum mechanics to generate the needed M-A vs $\mathrm{M}-\mathrm{AH}_{\mathrm{x}}, \mathrm{M}-\mathrm{CO}$ and other linear scaling relationships, there is a very serious initial cost associated with the large computational time needed for this[29]. We show below that the DRC method can circumvent some of these shortcomings in many situations when working with similar materials, but that the NB method is more robust to large changes in material type. 
The main question we address here is: How does the DRC method perform compared to the more commonly used NB method in comparing the rates of a variety of different catalysts when analyzing the same computational set of energies? We find that it works as well and even better (i.e., more accurately) than the NB method, at least for materials that are similar enough to the reference material that they have similar degrees of rate control (i.e., have descriptor energies within $0.3 \mathrm{eV}$ of the reference material). We also show that it gives qualitatively correct trends even for materials that differ by $\sim 1.3 \mathrm{eV}$ in their descriptor energies relative to the reference material, but can fail miserably when the materials are so different in electronic character that completely different species become most rate controlling (i.e., when descriptor energies differ by $>2 \mathrm{eV}$ from the reference material). Thus, it provides a complimentary tool to the NB method, but cannot replace it.

In order to test the practical applicability of this new method, we have chosen to use the methane steam reforming reaction as a test system. Recently, a great deal of attention and effort has been expended to discover and develop new methane reserves around the world using hydraulic fracturing technologies[30]. Thus, methane catalysis will only become more important in the foreseeable future, whether to produce hydrogen through steam reforming[31,32] or larger hydrocarbon molecules[33]. The performance and more importantly the cost of these catalysts will have a direct impact on how people chose to utilize these new methane reserves. It is also a system that has been thoroughly studied already by the NB approach described above[34,35], providing a convenient reference for comparison.

\section{Theory}

The NB Method and its Rate Volcano Calculations for Catalyst Screening

All rate volcanoes generated by the NB method were constructed using the Catalysis Microkinetic Analysis Package (CatMAP) module for Python,[36,37] which is a web-accessible program that essentially follows the procedure described above for the NB method, described in more detail in $[5,16]$. CatMAP allows for the calculation of catalytic rates, as well as the degree of rate control, given the standard-state free energies of all the adsorbed intermediates and transition states. DFT energies for the intermediates and transition states in methane steam reforming on all metals were taken from Xu et. al.,[35] while gas-phase formation energies were taken from NIST.[38] The heat capacities and entropic corrections to the standard-state free energies of adsorbates and transition-states are calculated within the harmonic oscillator 
approximation from vibrational and vibrational frequencies that are reported for $\mathrm{Rh}(211)$ by $\mathrm{Xu}$ et al[35]., and assuming these were the same for all metals. Entropy and heat capacity contributions of gas-phase molecules are derived using experimental values applying the Shomate equation[39]. The catalytic rates are calculated at steady-state using a mean-field kinetic model where lateral adsorbate-adsorbate interactions were neglected. It is important to note that the accuracy of the above approximations is not important for the main issue addressed in this paper, which is to compare the accuracy of the new DRC method for comparing rates on different materials with the NB method, when otherwise using the same exact approximations and underlying data to calculate rates.

The pseudo-steady-state approximation is employed to solve the resulting set of differential equations by assuming that the rate of change for all surface intermediates is zero. After obtaining the steady-state solution the degree of rate control is computed by numerical differentiation $\left(\delta \mathrm{E}=1 \times 10^{-12} \mathrm{eV}\right)$. All these methods are implemented in CatMap and more details can be found in.[36]

CatMAP allows for the computation of rates and the degrees of rate control either for any specific metal, as we do here for the reference material, $\operatorname{Rh}(211)$, or as a continuous function of the descriptor energies used for all the linear scaling relationships. It also generates the linear scaling relationships for each adsorbate and transition state using the DFT energies for a select set of materials. ${ }^{37}$

\section{The Degree of Rate Control Method for Catalyst Screening based on Activity}

The degree of rate control was defined in Eq. 1. We temporarily convert this from a partial derivative to a regular derivative for the special case where all the other parameters are constant, yielding

$$
X_{i}=\frac{d(\ln r)}{d\left(\frac{-G_{i}}{R T}\right)}
$$

Rearranging yields

$$
X_{i} * d\left(\frac{-G_{i}}{R T}\right)=d(\ln r)
$$


We can now integrate this over some change in $G_{i}$, from its value $G_{i, o}$ for some reference catalyst, denoted by a small $o$, to its value $G_{i, n}$ for some new catalyst, denoted by a small $n$. Assuming that $X_{i}$ remains constant over the range of interest, at constant temperature this gives:

$$
X_{i} *\left(\frac{-G_{i, n}-\left(-G_{i, o}\right)}{R T}\right)=\int_{r_{o}}^{r_{n}} d(\ln r)=\ln \frac{r_{n}}{r_{o}} .
$$

Equation (4) shows the change in the total rate to the product of interest, in moving from catalyst $o$ to catalyst $n$, due only to the change in the standard-state free energy of species i, $G_{i}$, and its degree of rate control $X_{i}$. This is only true if all other $X_{i}=0$ in the reaction. If some number $N$ species all have non-zero $X_{i}$, then the total change in rate is the sum of terms of identical form to the left-hand side of Eq. 4:.

$$
\sum_{i=1}^{N} X_{i} *\left(\frac{-G_{i, n}-\left(-G_{i, o}\right)}{R T}\right)=\ln \frac{r_{n}}{r_{o}} .
$$

Taking the exponent of both sides of the equation yields:

$$
\frac{r_{n}}{r_{o}}=e^{\sum_{i=1}^{N} X_{i} *\left(\frac{-G_{i, n}-\left(-G_{i, o}\right)}{R T}\right)} .
$$

We further assume that the standard-state entropy of the same adsorbed species or transition state is similar on different surfaces[27], so that the variations in standard-state free energy from surface to surface can be accurately approximated by changes in the electronic potential energy (i.e., the internal energy at $0 \mathrm{~K})$ computed using DFT $\left(E_{i, n}\right)$, so that:

$$
G_{i, n}-G_{i, o}=E_{i, n}-E_{i, o} .
$$

Thus, Eq. (7) simplifies to:

$$
\frac{r_{n}}{r_{o}}=e^{\sum_{i=1}^{N} X_{i} *\left(\frac{-E_{i, n}-\left(-E_{i, o}\right)}{R T}\right)} .
$$

Equation (8) allows one to estimate $r_{n} / r_{o}$ for new but similar materials to the reference material, given the internal energies for both materials of all $\mathrm{N}$ intermediates and transition states that have non-negligible degrees of rate control at the conditions of interest. In general, $\mathrm{N}$ is a very small fraction of the total number of species in the mechanism[22-26]. Here, their internal energies will be calculated by DFT, but in principle these could be estimated by any other electronic structure method, by semi-empirical methods or by experimental measurements.

We show below that Eq. (8) can be used to computationally search for new catalyst materials which possesses higher activity than the reference material (i.e., $r_{n} / r_{o}>1$ ), and sort amongst many of these to predict the best materials. It assumes that the DRCs (i.e., the $X_{i}$ values 
that are large enough to matter) for these other materials are the same as the reference material at the chosen conditions. We show below the limitations of this assumption: It is accurate for materials that have similar electronic character to the reference material, but fails when the materials are very different. To summarize, the steps of this "DRC method" for catalyst screening are:

1-2. Follow steps 1) and 2) from the NB method (see Introduction), or briefly: Determine the reaction's mechanism and active site on the reference material, and develop a microkinetic model for that mechanism.

3. Calculate the degrees of rate control for all adsorbates and transition states in the microkinetic model on the reference material at the reaction conditions of interest. (We discuss below an experimental method that allows one to bypass Steps 1-2 to get the needed DRCs.)

4. For all adsorbates and transition states with large degrees of rate control, calculate the internal energies of those species on a new potential catalyst material using DFT.

5. Calculate the relative rate for that new catalyst using Eq. 8 and the DRCs and energies for the reference catalyst along with the energies for the new potential catalysts.

6. Repeat steps 4-5 for each new material to be screened, and generate a plot of rate versus the energies of the species with large DRCs. This would typically be a two- to four-dimensional plot.

We will refer to this as the "degree of rate control method" or DRC method.

This method relies on using a reference catalyst and assuming that the DRCs (coverages) of adsorbates are similar to that reference material on the materials being screened. While we do not do so in the present paper, one can easily include adsorbate-adsorbate interactions into this method by simply calculating the needed adsorption energies for adsorbates that have large DRCs (i.e. high coverages) at the coverages found for the reference material at the reaction conditions of interest. The same is true for the energies for any transition states with large DRCs that might depend on adsorbate coverages: use the coverages of the reference material to get those transition state energies. The DRC method is quite general and should be applicable to any chosen reaction conditions and mechanism, including mechanisms where steps occur on different sites or facets, as in the mechanism used below to demonstrate the method. The limitations of its 
accuracy compared to experiments are the same as those associated with the quantum mechanical method used (like DFT) and the assumption that the DRC values that are large are the same as on the reference material.

This DRC method has also been implemented in the CatMAP software, providing an automated approach to optimizing a catalyst once the degrees of rate control are known.

In some cases it is possible to estimate the degrees of rate control for the species with large DRCs via experimental measurements (see below), allowing one to skip steps 1-3 above, and search for better materials without developing a full microkinetic model for the reference material, or even knowing the complete mechanism.

\section{The Degree of Rate Control Method for Catalyst Screening based on Selectivity}

In searching for better catalysts, one is often more concerned with improving selectivity than activity. It is trivial to extend the above DRC approach to focus on selectivity instead of activity, since the DRC can be defined relative to the rate for making any product or for consuming any reactant. For example, let $\mathrm{P}$ be the most desired product and $\mathrm{R}$ be the most expensive reactant, with $r_{P}$ the rate to $\mathrm{P}$ and $r_{R}$ the rate of consumption of $\mathrm{R}$, with the selectivity then being $\mathrm{S}=r_{P} / r_{R}$. Let $X_{P i}$ and $X_{R i}$ be the corresponding degrees of rate control of these two rates for species i. One can write down the equivalent of Eq. (5) for both these rates:

$$
\ln \frac{r P_{n}}{r_{P o}}=\sum_{i=1}^{N} X_{P i} *\left(\frac{-G_{i, n}-\left(-G_{i, o}\right)}{R T}\right)
$$

and

$$
\ln \frac{r R_{n}}{r_{R o}}=\sum_{i=1}^{N} X_{R i} *\left(\frac{-G_{i, n}-\left(-G_{i, o}\right)}{R T}\right)
$$

Subtracting these two equations gives:

$$
\ln \frac{r P_{n}}{r_{P o}}-\ln \frac{r R_{n}}{r_{R o}}=\sum_{i=1}^{N}\left(X_{P i}-X_{R i}\right) *\left(\frac{-G_{i, n}-\left(-G_{i, o}\right)}{R T}\right) .
$$

Rearranging gives a relationship for the selectivity ratio of the new material to the reference:

$$
\ln \frac{r P_{n}}{r_{P o}}-\ln \frac{r R_{n}}{r_{R o}}=\ln \frac{r_{\frac{P n}{2}}}{r_{R o}} \frac{\operatorname{ros}}{r_{R o}}=\ln \frac{S_{n}}{S_{o}}=\sum_{i=1}^{N}\left(X_{P i}-X_{R i}\right) *\left(\frac{-G_{i, n}-\left(-G_{i, o}\right)}{R T}\right) .
$$


Applying the same steps as used between Eq. (5) to (8) above gives the selectivity ratio equivalent of the activity ratio in Eq. (8) above:

$$
\frac{S_{n}}{S_{o}}=e^{\sum_{i=1}^{N}\left(X_{P i}-X_{R i}\right) *\left(\frac{-E_{i, n}-\left(-E_{i, o}\right)}{R T}\right)} .
$$

Notice the close similarity to the right side of Eq. (8): $X_{i}$ is simply replaced with $X_{P i} X_{R i}$. Thus, Eq. (8-S) is just as easy to apply as Eq. (8) except that it requires two degrees of rate control for each transition state and intermediate instead of one: one for the rate of making $\mathrm{P}$ and one for the rate of consuming R. Although its use is not demonstrated in this paper, our proof below of the accuracy and simplicity of Eq. (8) in searching for catalysts with better activity implies that Eq. (8-S) will be useful in searching for catalysts with better selectivity, and almost as fast as screening based on activity. As with Eq. (8), Eq. (8-S) assumes that the steps with large DRCs have the same DRC values on the new material as on the reference material. We show below that this assumption is reasonably accurate for materials that have similar electronic character to the reference material, but it fails when the materials are too different. Because Eq. (8-S) uses two DRC values per species but Eq. (8) only uses one, Eq. (8-S) is expected to fail sooner in this respect.

The concept of "degree of selectivity control" was introduced by Campbell et al. ${ }^{19}$.

\section{Results and Discussion}

Catalyst screening was performed with both methods at the following methane steam reforming reaction conditions: $\mathrm{T}=773 \mathrm{~K}, \mathrm{P}_{\mathrm{CO}}=0.05$ bar, $\mathrm{P}_{\mathrm{H} 2}=0.15$ bar, $\mathrm{P}_{\mathrm{CH} 4}=\mathrm{P}_{\mathrm{H} 2 \mathrm{O}}=0.40$ bar, and comparison was made on the basis of the steady-state TOF of CO production. We used the same mechanism as used by Xu et al.[35] in their previous study of this system using the NB method. Just as they did, we calculate the rates only for the (211) faces of the different metals, since they found that the (211) surface is mainly responsible for the rate. This mechanism is given below, where $\mathrm{a} *$ symbol refers to a surface site:

(a) $\quad \mathrm{CH}_{4(\mathrm{~g})}+*^{\mathrm{t}}+*^{\mathrm{h}} \rightleftharpoons \mathrm{CH}_{3} *^{\mathrm{t}}+\mathrm{H}^{* \mathrm{~h}}$,

(b) $\mathrm{CH}_{3} *^{\mathrm{t}}+*^{\mathrm{h}} \rightleftharpoons \mathrm{CH}_{2} *^{\mathrm{t}}+\mathrm{H}^{* \mathrm{~h}}$,

(c) $\mathrm{CH}_{2} *^{\mathrm{t}}+*^{\mathrm{h}}+*^{\mathrm{f}} \rightleftharpoons \mathrm{CH}^{* \mathrm{f}}+\mathrm{H}^{* \mathrm{~h}}+*^{\mathrm{t}}$,

(d) $\mathrm{CH}^{* \mathrm{f}}+*^{\mathrm{h}} \rightleftharpoons \mathrm{C}^{* \mathrm{f}}+\mathrm{H}^{* \mathrm{~h}}$,

(e) $\mathrm{H}_{2} \mathrm{O}_{(\mathrm{g})}+*^{\mathrm{s}}+*^{\mathrm{h}} \rightleftharpoons \mathrm{OH}^{* \mathrm{~s}}+\mathrm{H}^{* \mathrm{~h}}$,

(f) $\mathrm{OH}^{* \mathrm{~s}}+*^{\mathrm{h}} \rightleftharpoons \mathrm{O}^{* \mathrm{~s}}+\mathrm{H}^{* \mathrm{~h}}$, 


$$
\mathrm{C}^{*^{\mathrm{f}}}+\mathrm{OH}^{* \mathrm{~s}} \rightleftharpoons \mathrm{COH}^{* \mathrm{~s}}+*^{\mathrm{f}}
$$

(h) $\mathrm{COH}^{* \mathrm{~s}}+*^{\mathrm{h}} \rightleftharpoons \mathrm{CO}^{*^{\mathrm{s}}}+\mathrm{H}^{* \mathrm{~h}}$,

(i) $\mathrm{CH}^{* \mathrm{f}}+\mathrm{O}^{* \mathrm{~s}} \rightleftharpoons \mathrm{CHO}^{* \mathrm{~s}}+*^{\mathrm{f}}$,

(j) $\mathrm{CHO}^{* \mathrm{~s}}+*^{\mathrm{h}} \rightleftharpoons \mathrm{CO}^{* \mathrm{~s}}+\mathrm{H}^{* \mathrm{~h}}$,

(k) $\quad \mathrm{C}^{* \mathrm{f}}+\mathrm{O}^{* \mathrm{~s}} \rightleftharpoons \mathrm{CO}^{* \mathrm{~s}}+*^{\mathrm{f}}$,

(l) $\mathrm{CO}^{* \mathrm{~s}} \rightleftharpoons \mathrm{CO}_{(\mathrm{g})}+*^{\mathrm{s}}$,

(m) $\quad 2 \mathrm{H}^{* \mathrm{~h}} \rightleftharpoons \mathrm{H}_{2(\mathrm{~g})}+2^{* \mathrm{~h}}$.

These adsorbates may be adsorbed on one of four different sites on the (211) face on the metal: The 'step' (*s) site corresponds to the upper part of a (211) step site. The 'four-fold' $\left({ }^{*} \mathrm{f}\right)$ site corresponds to the lower four-fold site of a (211) step. The 'terrace' $\left({ }^{*} t\right)$ corresponds to a (211) terrace site. In addition, hydrogen is adsorbed in a special 'hydrogen reservoir' site $\left({ }^{*} \mathrm{~h}\right)$ which has the same energetics as a three-fold hollow site at steps. These four site types are considered as independent of each other, so that species on one type of site do not block sites in any way for the adsorption of species on another type of site.

Since the production of $\mathrm{CO}_{2}$ (from $\mathrm{CO}^{*}+\mathrm{O}^{*}$ ) was not considered in the original modelling of this reaction's rate by the NB method[35], we have not included it in this mechanism. It is not necessary for comparing these different theoretical methods for estimating rates within a given mechanism, which is our focus here. The mechanism as used here is sufficiently complex to allow for a good comparison of the relative merits of these methods.

We selected $\mathrm{Rh}(211)$ as the "reference" catalyst because it showed one of the highest TOFs at these conditions, but also because, in order for us to do the DRC analysis, it was necessary to have DFT energies for all intermediates and transition states, and Rh was a metal for which all these energies were readily available[35,40,41].

\section{Catalyst Screening by the NB Method}

Here we followed essentially the same procedure as used by Xu et al.[35], and obtained very similar results for the rates on the (211) facets of different metals. The energies of surface $\mathrm{C}$ and $\mathrm{O}$ atoms $\left(E_{C^{*}}\right.$ and $\left.E_{O^{*}}\right)$ are the descriptors for the linear scaling relations. That is, the energies of all the adsorbates and intermediates were assumed to be linear combinations of $E_{C^{*}}$ and $E_{O^{*}}$. The steam reforming volcano plot of rate as a function of these two descriptors is shown in Fig. 1a. It is very similar to that reported by Xu et al.[35], with minor discrepancies that arise due to their different treatments of the standard-state entropies and heat capacities for 
adsorbed species as implemented in CatMap. In both models, standard-state entropies and heat capacities for adsorbates and transition states were calculated assuming localized adsorbates and the harmonic oscillator approximation together with the vibrational frequencies reported for $\mathrm{Rh}(211)$ by $\mathrm{Xu}$ et al.[35], assuming these same frequencies apply to all metals. In the present work, this was done by explicitly integrating up to $773 \mathrm{~K}$, whereas $\mathrm{Xu}$ et al. did this from $0 \mathrm{~K}$ to $300 \mathrm{~K}$, and assumed that the entropy and heat capacity were constant at the $300 \mathrm{~K}$ value between $300 \mathrm{~K}$ and $773 \mathrm{~K}$. The NB rate volcano was derived using scaling relations to estimate the energies of all adsorbates and transition states. This is an approximation, as the actual DFT values for the transition-metals are spread around the scaling lines with a standard deviation of $0.20 \mathrm{eV}$ for the adsorbates and transition states of the microkinetic model laid out by $\mathrm{Xu}$ et al.[35] and repeated earlier in this paper.

The scaling approximations of the NB method result in some error in the resulting rates, as shown in Fig. 1b, which compares the rates (relative to $\mathrm{Rh}(211)$ ) computed using the DFT energies directly with the rates computed with the energies estimated from the linear scaling relationships (i.e. the rates that appear on the volcano in Fig. 1a) for some metals near $\mathrm{Rh}(211)$ in Fig. 1a. It is clear that although the general trend in rates with metals is similar for both, there are some significant quantitative errors (e.g. the rate on Ir is over-estimated by $>2$ orders of magnitude using the scaling relations, and the standard deviation of the points about the perfectagreement line here is 1.48). Note that we compare two theoretically derived values here. Thus, we do not know which of the values is more accurate relative to reality for a given metal. However, since the linear scaling values are derived from the DFT values as an approximation to them, they should be considered less accurate in general. This will certainly be the case as more accurate DFT functionals are developed. Also, the main goal of this paper is to compare methods for computationally screening catalysts, assuming that one has an accurate method for calculating energies. The conclusions one draws in this comparison by assuming that the DFT method used here is perfectly accurate will remain valid as DFT methods gain accuracy. 

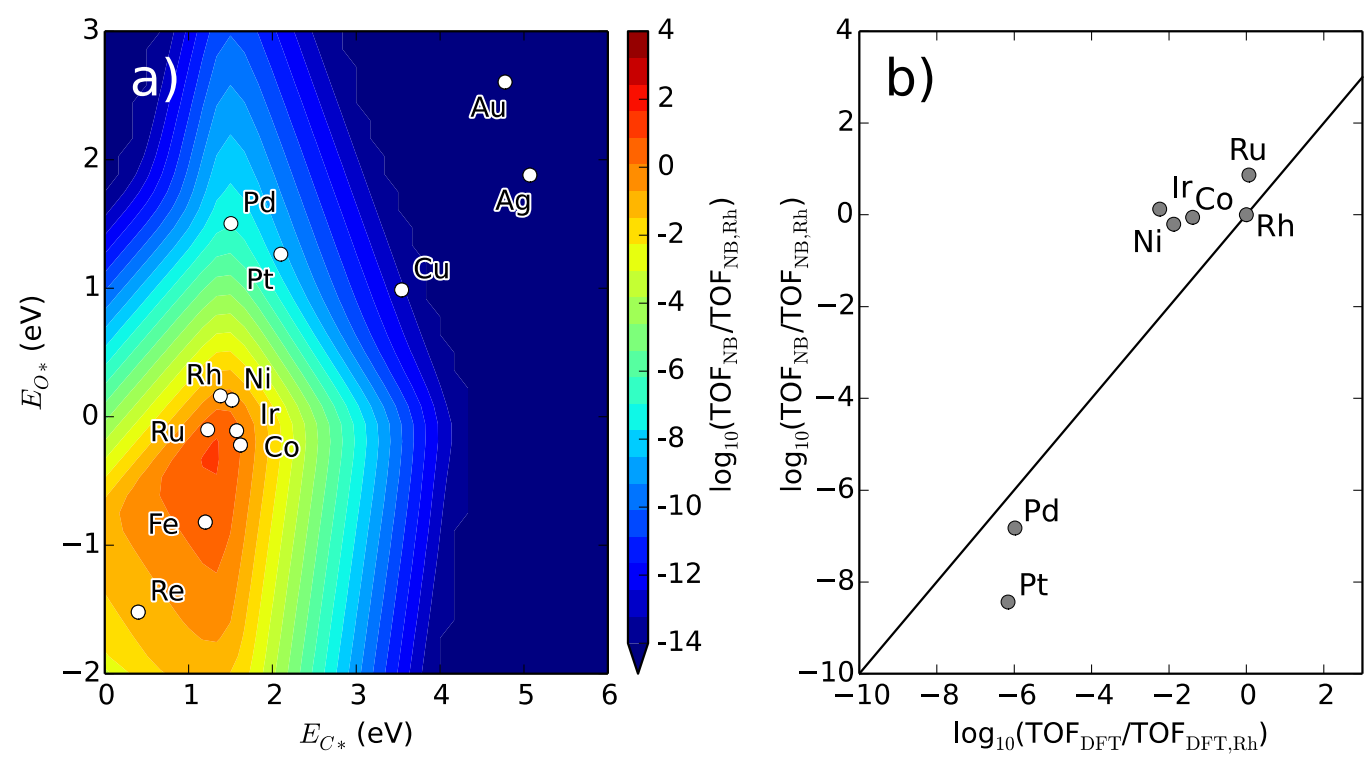

Figure 1. (a) Volcano plot of catalyst activity (log TOF) for steam reforming of methane on the (211) facets of different metals calculated by the NB method plotted as a function of $\mathrm{C}^{*}$ and $\mathrm{O}^{*}$ adsorption energy (defined here as the energies for the reactions $\mathrm{CH}_{4(\mathrm{~g})} \rightarrow \mathrm{C}^{*}+2 \mathrm{H}_{2(\mathrm{~g})}$ and $\mathrm{H}_{2} \mathrm{O}_{(\mathrm{g})} \rightarrow$ $\mathrm{O}^{*}+\mathrm{H}_{2(\mathrm{~g})}$, respectively). Energies of other intermediates are estimated using linear scaling relations. Reaction conditions are $\mathrm{T}=773 \mathrm{~K}, \mathrm{P}_{\mathrm{CO}}=0.050$ bar, $\mathrm{P}_{\mathrm{H} 2}=0.15$ bar, $\mathrm{P}_{\mathrm{CH} 4}=\mathrm{P}_{\mathrm{H} 2 \mathrm{O}}=0.40$ bar. The TOFs are normalized to the TOFs on $\mathrm{Rh}$ in order to more easily compare to the DRC method later.

(b) Comparison of these rates computed by the NB method using linear scaling relations with those calculated directly with DFT energies, which are considered the "true" rates for the purpose of this paper, i.e., to compare these "true" DFT rates with rates from faster methods which simply estimate them.

\section{Catalyst Screening Using the Degree of Rate Control Method}

The degree of rate control for each intermediate and transition-state was computed for methane steam reforming at these same conditions on $\mathrm{Rh}(211)$ using DFT energies directly (as opposed to linearly scaled energies). The three species with the highest degrees of rate control for $\mathrm{Rh}(211)$ were found to be the transition state for the formation of adsorbed $\mathrm{CO}\left(\mathrm{X}_{\mathrm{C}-\mathrm{O}^{*}}=\right.$ $0.87)$, the adsorbed $\mathrm{CO}$ intermediate $\left(\mathrm{X}_{\mathrm{CO} *}=-0.76\right)$, and adsorbed intermediate of atomic carbon $\left(\mathrm{X}_{\mathrm{C}^{*}}=-0.54\right)$. A complete list of the degree of rate control for all species is given in Table 1. Note that in this mechanism, some intermediate states include several adsorbates. However, in this model the energies of all adsorbates are independent of each other and the energies of such 
intermediates are their simple sums, so that the partial derivatives with respect to the energy of an intermediate state can be further subdivided into partial derivatives with respect to the energies of individual adsorbates. We have done that here.

Table 1. The degrees of rate control for all intermediates and transition states for $\mathrm{Rh}(211)$ at the same conditions as Fig. 1.

\begin{tabular}{|c|c|c|c|}
\hline Species & $X_{i}$ & Species & $X_{i}$ \\
\hline \multicolumn{2}{|c|}{ Intermediates } & \multicolumn{2}{|c|}{ Transition States } \\
\hline $\mathrm{CO}^{* \mathrm{~s}}$ & -0.76 & $\mathrm{C}-\mathrm{O}^{*}$ & 0.87 \\
\hline$C^{* f}$ & -0.54 & $\mathrm{H}-\mathrm{CH} 3^{*}$ & 0.11 \\
\hline $\mathrm{CH}^{* \mathrm{f}}$ & -0.01 & $\mathrm{H}-\mathrm{OH}^{*}$ & 0.01 \\
\hline $\mathrm{H}^{* h}$ & -0.01 & $\mathrm{CO}-\mathrm{H}^{*}$ & 0.00 \\
\hline $0^{* 5}$ & 0.00 & $\mathrm{H}-\mathrm{C}^{*}$ & 0.00 \\
\hline $\mathrm{OH}^{* \mathrm{~s}}$ & 0.00 & $\mathrm{H}-\mathrm{CH}^{*}$ & 0.00 \\
\hline $\mathrm{CH}^{*} *^{\mathrm{t}}$ & 0.00 & $\mathrm{H}-\mathrm{CH} 2^{*}$ & 0.00 \\
\hline $\mathrm{CH} 2^{* t}$ & 0.00 & H-CO* & 0.00 \\
\hline $\mathrm{HCO}^{* \mathrm{~s}}$ & 0.00 & C-OH* & 0.00 \\
\hline $\mathrm{COH}^{* \mathrm{~s}}$ & 0.00 & O-CH* & 0.00 \\
\hline & & $\mathrm{H}-\mathrm{H}^{*}$ & 0.00 \\
\hline & & O-H* & 0.00 \\
\hline
\end{tabular}

It is well known that the $\boldsymbol{X}_{\boldsymbol{i}}$ values of some species depend sensitively on reaction conditions[22], so the values listed here are specific to the reaction conditions being modelled. The $\boldsymbol{X}_{\boldsymbol{i}}$ values for the two intermediates $\mathrm{CO}^{* \mathrm{~s}}$ and $\mathrm{C}^{* \mathrm{f}}$ sum to an absolute magnitude greater than one here because they sit on different sites and do not block each other. The absolute magnitudes of the $\boldsymbol{X}_{\boldsymbol{i}}$ values for each site must sum to a maximum of 1 (or some small integer[13]) or less, but with different sites they can sum to greater than 1. Since H uses separate sites than all other adsorbates, its DRC equals 0 in this model. The large $X_{i}$ for the transition state for the step $\mathrm{C}^{* \mathrm{f}}+\mathrm{O}^{* \mathrm{~s}} \rightarrow \mathrm{CO}^{* \mathrm{~s}}$ shows that it is nearly the rate determining step in this model on $\mathrm{Rh}(211)$. It is even slower to produce $\mathrm{CO}^{* \mathrm{~s}}$ from either $\mathrm{COH}^{* \mathrm{~s}}$ or $\mathrm{CHO}^{* \mathrm{~s}}$ dissociation via Steps $(\mathrm{g})+(\mathrm{h})$ or $(\mathrm{i})+(\mathrm{j})$, respectively, so that almost all the $\mathrm{CO}^{* \mathrm{~s}}$ is produced via the step $\mathrm{C}^{* \mathrm{f}}+$ $\mathrm{O}^{* \mathrm{~s}} \rightarrow \mathrm{CO}^{* \mathrm{~s}}$ at these reaction conditions, as has been reported earlier [35]. Note that the $\boldsymbol{X}_{\boldsymbol{i}}$ values in Table 1 might not be in agreement with experiments, since DFT has some energy errors. However, that difference would not affect the conclusions of this paper about the relative 
merits of the DRC method compared to the NB method for computational screening of catalyst materials, since for the sake of that comparison, the rates calculated from the DFT energies for all the species must be considered to be the "true" rates. Comparison of these DFT and related NB results to experiments is discussed in the original paper[35].

The DRC method assumes that all the $X_{i}$ values for other materials (in this case, the (211) faces of all other metals) are equal to their values on the reference material, $\operatorname{Rh}(211)$, and estimates the rates of these other catalysts relative to $\mathrm{Rh}(211)$ using Eq. (8) together with the appropriate DFT energies. The sum in Eq. (8) really runs only over four species at most, since $X_{i}$ is negligibly small for all of the other 18 species, as seen in Table 1 . We will present the results of this four-species approach below, but let us first simplify even further by using only the two species with the largest DRCs, which will allow us to make a two-dimensional plot like Fig. $1 \mathrm{~b}$.

Assuming that all $X_{i}$ equal 0 except the two dominating species with $/ X_{i} />0.75$, one can directly estimate the rates of other catalysts relative to Rh using Eq. (8) together with the energies of these two species, $\mathrm{CO}^{*}$ and $\mathrm{C}-\mathrm{O}^{*}$, as the two descriptors that define all other materials. The results are shown in Fig. 2a as a contour plot. Figure 2 also shows distinct points for eleven of the other specific metal catalysts studied in the report of Xu et al.[35], using the DFT energies of the CO* and C-O* species for these different metal (211) surfaces. Fig. 2b shows that the rates estimated by DRC method for the metals similar to $\mathrm{Rh}(211)$ are in excellent agreement with the results from the actual DFT calculations and microkinetic model. Indeed, that agreement shows a significant improvement when comparing actual DFT numbers and the rates obtained by the NB scaling method, with a standard deviation from the straight line in Fig. $2 \mathrm{~b}$ of 1.22 compared to 1.48 for the NB method in Fig. 1b. In particular, the rate of Ir is in much better agreement due to the fact that the key energies, $\mathrm{E}_{\mathrm{CO}}$ and $\mathrm{E}_{\mathrm{C}-\mathrm{O}}$, are taken directly from DFT rather than estimated with linear scaling relations. 

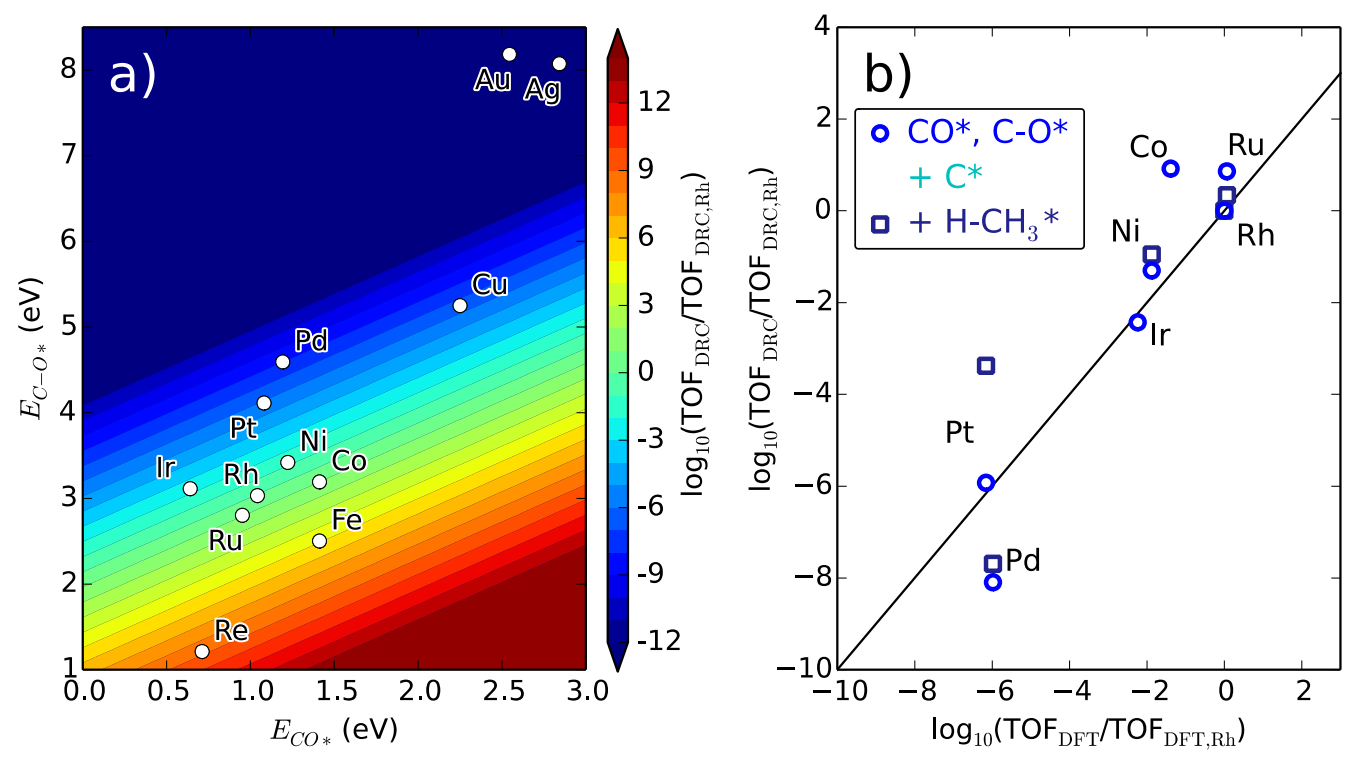

Figure 2. (a) Rates of methane steam reforming estimated for the (211) facets of different metals calculated using the DRC method using the DFT energies of the two species with the highest decrees of rate control as the only descriptors: the adsorption energy of $\mathrm{CO}^{*}$ and the transition-state energy for $\mathrm{CO}$ dissociation (the two species in the reaction mechanism with the highest degrees of rate control). All other species were assumed to have negligible DRCs.

(b) A comparison of these DRC-estimated rates to the rates computed with a full microkinetic model and DFT energetics. Rates estimated using the DRCs for $\mathrm{CO}^{*}$ and $\mathrm{C}-\mathrm{O} *$ only are shown in blue (circles). The effect of adding the DRC for $\mathrm{C}^{*}$ is shown in cyan (triangles), while adding DRCs for both $\mathrm{C}^{*}$ and $\mathrm{H}$ $\mathrm{CH}_{3} *$ is shown in navy (squares). (Since the DFT energy of $\mathrm{H}_{-} \mathrm{CH}_{3} *$ was not known for some metals, those metals have no square points.)

The rates estimated in Fig. 2a represent a partial implementation of the DRC method since the DRCs of only two species are included here, but there are four species with non-negligible DRC's (see Table 1). Visualization of rates depending on more than two dimensions is difficult. However, it is possible to compute the rates using all relevant DRCs for each metal. The effect of adding a third and fourth DRC are shown in Fig. $2 b$ (triangles and squares, respectively). The inclusion of the DRC for $\mathrm{C}^{*}$ makes a significant difference for several of the rates, while further addition of the DRC for $\mathrm{H}_{-} \mathrm{CH}_{3}$ * has a negligible impact due to its relatively small DRC. Surprisingly, the additions of the third and fourth DRCs $\left(\mathrm{C}^{*}\right.$ and then $\left.\mathrm{H}_{-}-\mathrm{CH}_{3} *\right)$ slightly decrease the accuracy of the method compared to direct DFT rates (standard deviation relative to the 
straight line of 1.54 and 1.45 , compared to 1.22 with just two DRCs). This is due to the assumption that the DRC is constant throughout the region of interest, but it changes for $\mathrm{C}^{*}$ when going to some of these metals (see below).

\section{Comparison of Screening Approaches}

\section{Predicted Catalytic Activity}

The DRC method exhibits improved accuracy over the NB rate volcano method for metals closest to the reference material (Fig. $1 b$ and $2 b$ ), but the fact that the DRC method does not exhibit an optimum places it at odds with the Sabatier principle, indicating that the improved accuracy will not hold globally. In general, it is expected that the DRC method will become less accurate as the materials become less similar to the reference material, since in that case it is less likely that the key DRCs continue to have similar values to the reference material.

One method for quantifying this difference is the distance in the $\left(E_{C^{*}}, E_{O^{*}}\right)$ plane from that reference material. The rates for the 2D, 3D, and 4D DRC method are shown in Fig. 3 as a function of this distance, along with the results from direct DFT calculations in the full microkinetic model (DFT+MKM) and the results from the NB method. Figure 3 demonstrates that when the distance is $<0.5 \mathrm{eV}$, the results agree relatively well, and the DRC method is even slightly more accurate, while beyond $0.5 \mathrm{eV}$ there are significant quantitative and even qualitative differences. When the materials are very different from the reference catalyst $(>1.5$ $\mathrm{eV}$ in NB descriptor space, i.e., on a plot where the axes are the bond energies to $\mathrm{C}$ and $\mathrm{O}$ adatoms as in Fig. 1a), the DRC method is much less accurate than the NB method. Since better catalysts have almost always been found computationally by the NB method to be within the group of materials that have similar descriptors to the best reference material (i.e., within $0.5 \mathrm{eV}$ in NB descriptor space)[5,8-10,12,42-48], this is not a serious limitation. Still, the NB method is clearly much more robust than the DRC method to large changes in material type. As discussed below, in principle one could also maintain the accuracy of the DRC approach to more dissimilar materials by moving in steps of $<0.5 \mathrm{eV}$ to a better material, and then recalculating the DRCs for that new reference material, and move further from there. However, it remains to be demonstrated how well this would work, and it would likely have extra difficulties if one tried to move between completely different material types, as for example from metals to metal oxides. It is likely that the mechanism and sites would also change with such a large change in material 
type, in which case a very basic and important assumption of both this DRC method and the NB method would fail. The same failure would occur if the material restructured or oxidized under reaction conditions.

We noted above that Eq. (8-S) could be used to search for more selective catalysts via the DRC method. It is not yet clear whether more selective new catalysts will also usually be found to be relatively close to the most selective known reference catalyst(in NB descriptor space). Evidence proves that they are sometimes found by the NB method to be in much more distant regions.[40] The DRC approach will therefore often prove less useful than the NB method in those cases.

In order to understand the reason for these changes in accuracy in the DRC method moving to the right in Fig. 3, one must think about how the degree of rate control for an intermediate (in this case $\mathrm{CO}^{*}$ ) and the coverage of that intermediate are related. The absolute magnitude of $\mathrm{X}_{\mathrm{CO}}(0.87)$ just equals $\theta_{C O}$ here, as expected ${ }^{14}$, and the $\mathrm{CO}$ coverage is still low enough to change sensitively with $\mathrm{CO}$ adsorption energy if it is decreased by much. For example, if we assume its coverage is determined by simple first-order adsorption/desorption equilibrium, $\theta_{C O}$ will decrease from 0.87 by a factor of ten for every decrease in its desorption energy (increase in $\mathrm{E}_{\mathrm{CO}}$ ) of $0.15 \mathrm{eV}=\mathrm{kT} \ln 10$, after the first factor of ten, which requires a larger $(\sim 0.3 \mathrm{eV})$ decrease due to the effect of near saturation in CO initially. Due to this, the large increase in rate due to such increases in $\mathrm{E}_{\mathrm{CO}}$ (i.e., moving far to the right along the $\mathrm{E}_{\mathrm{CO}}$ axis in Fig. 2) predicted by Eq.(8) will not be nearly as strong as expected, since $\mathrm{X}_{\mathrm{CO}}$ * will soon start to decrease rapidly as well. This effect of moving to the left here on $\mathrm{X}_{\mathrm{CO}} *$ is almost negligible however, since the $\mathrm{CO}$ coverage of 0.87 is almost saturated and its DRC can increase at most to 1.0 (i.e., change by <15\%). Thus, the predictions of Eq. (8) will remain much more accurate when moving to the left in Fig. 2 than when moving to the right. It is also worth pointing out that when extending this model to include the three highest DRC species, the coverage of $\mathrm{C}^{*}$ is also equal to its DRC (-0.54) because it adsorbs on a different type of site than CO*. This type of analysis provides an a priori route to estimating the region where the DRC method will be accurate. 


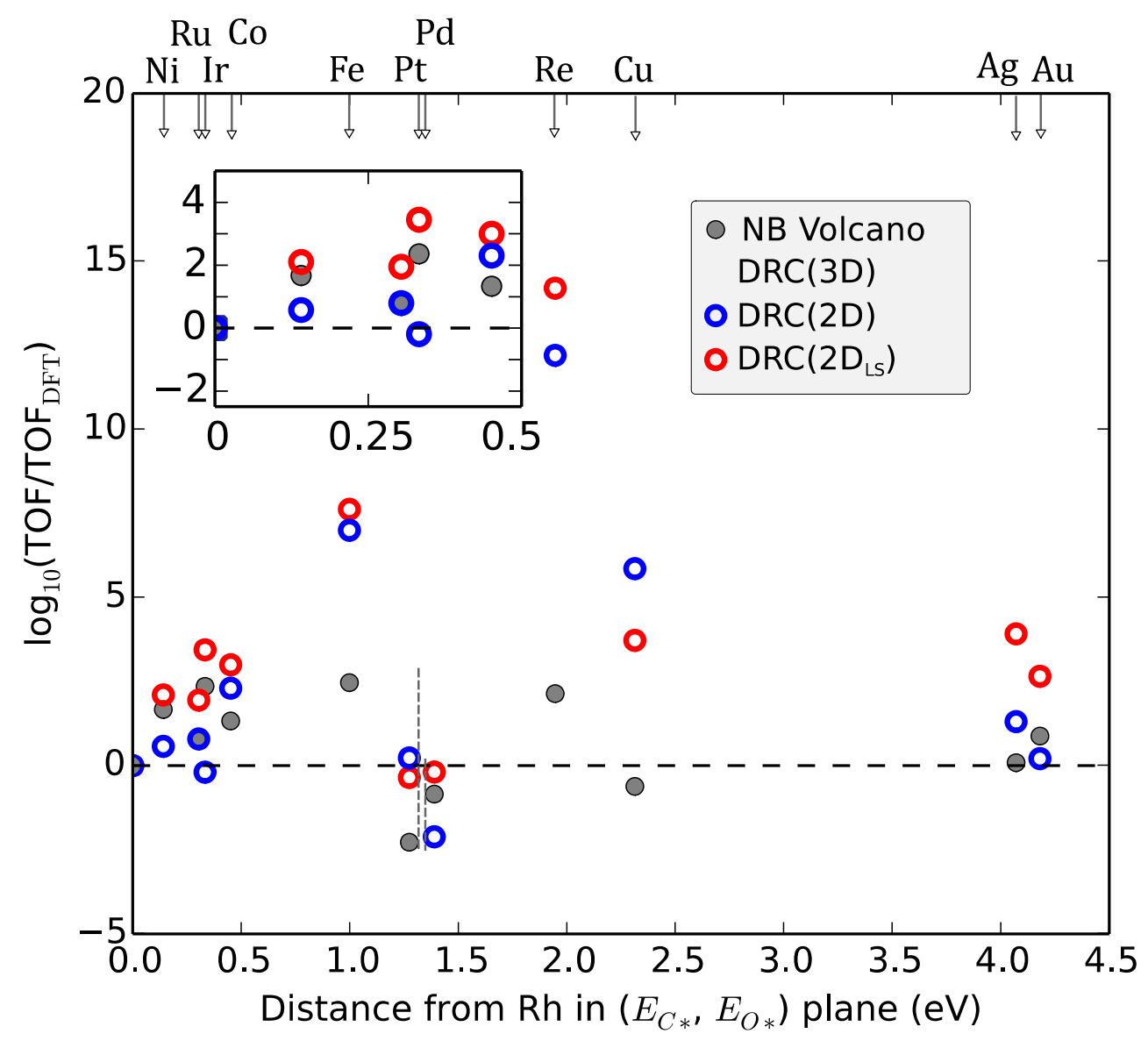

Figure 3: A comparison of the calculated TOFs relative to $\mathrm{Rh}(211)$ for the (211) facets of different metals as a function of distance from $\mathrm{Rh}$ on the $\left(E_{C^{*},} E_{O^{*}}\right)$ plane of Fig. 1a. Symbols refer to different methods, as given in the legend. DRC(2D) and DRC(3D) refer to the number of species used in the DRC method. The DRC(4D) points (not shown) are very close to the DRC(3D) points. Data are the same as in Figs. 1a and 2a. The DRC $\left(2 \mathrm{D}_{\mathrm{LS}}\right)$ method (red circles) uses linear scaling relations to estimate all the inputs to the DRC method, and is shown in Fig. 4a. The inset magnifies the region below $0.5 \mathrm{eV}$ from $\mathrm{Rh}$, where the DRC method is expected to be most accurate. The $\mathrm{X}$-axis positions for $\mathrm{Pt}$ and $\mathrm{Pd}$ have been separated slightly in order to more clearly differentiate their points.

Another route to visualizing the accuracy of the DRC method is to compare it directly to the NB rate volcano in Fig. 1a. In order to show this comparison approximately, the DRC method was 
calculated using energies from the linear scaling relations (LS-DRC) (rather than directly from DFT as in Fig. 2). This allows us to compute the DRC rate as a function of $\mathrm{C}^{*}$ and $\mathrm{O}^{*}$ for direct comparison to the results from the NB method of Fig. 1. The resulting DRC rate is shown in Fig. $4 \mathrm{a}$, using here only the two species with highest DRC's (CO* and C-O*) and compared directly to the rates from the NB method in Fig. 4b. The difference DRC plot shows that the DRC method is very accurate near $\mathrm{Rh}$, but becomes highly inaccurate at long distances in the $\left(E_{C^{*}}\right.$, $\left.E_{O^{*}}\right)$ plane. This is due to the violation of the constant DRC assumption that is used in applying Eq. (8).
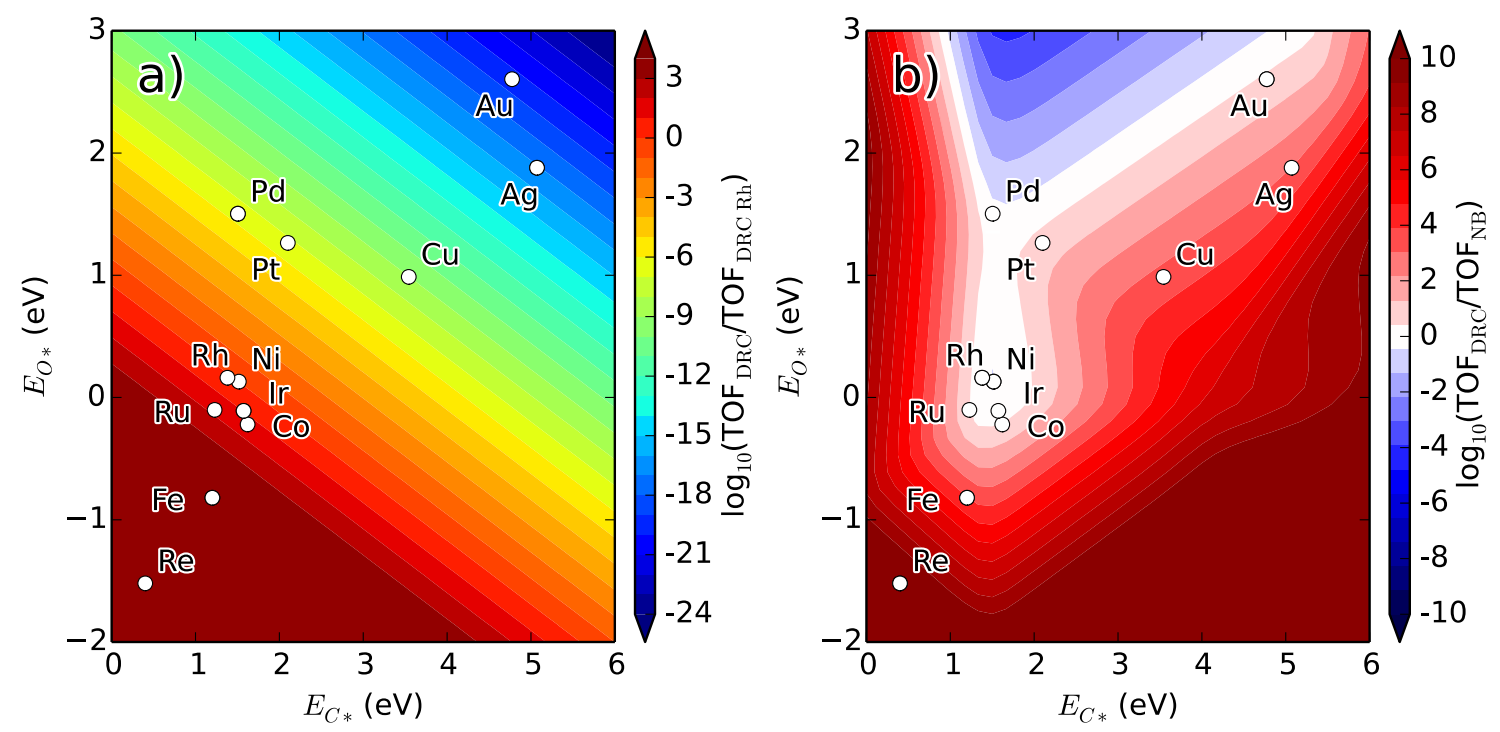

Figure 4: (a) Rates on the (211) facets of different metals computed with the DRC method assuming only the two species with the highest DRCs on $\mathrm{Rh}(211)\left(\mathrm{C}-\mathrm{O}^{*}\right.$ and $\mathrm{CO}^{*}$ ) have nonnegligible DRCs, but now modified from Fig. 2a by using linear scaling relations to estimate the energies of these two descriptors, rather than using DFT energies directly. Conditions are the same as Fig 1.

(b) The ratio of these rates to rates from the NB method of Fig. 1a.

In order to better illustrate the validity of the constant DRC assumption, the degrees of rate control for adsorbed species and transition states are shown in Fig. 5. It is clear that the degree of rate control changes drastically throughout the $\left(E_{C^{*}}, E_{O^{*}}\right)$ plane; however, there are large sections where the degrees of rate control are constant. The fact that $\mathrm{Rh}$ falls on the border of several such 
sections for the steam reforming reaction indicates that this example provides a very rigorous test of the accuracy of the DRC method. For reaction conditions and catalysts where the degree of rate control is dominated by one transition state and one intermediate, as is often the case and is clearly the case in several large regions of these figures where the color is the same, the DRC method will remain accurate for a wider range of materials, since its assumptions remains valid over a wider range. Still, the best known catalyst for any given reaction will often be like $\mathrm{Rh}$ is in this reaction: close to the boundary between such regions when DRCs change more rapidly.

To partially bypass the problem of changing DRC values for the key species, one could also adapt the simple DRC method applied here by moving only small distances in the $\left(\mathrm{E}_{\left.\mathrm{C}^{*}, \mathrm{E}_{\mathrm{O}^{*}}\right)}\right.$ plane using constant DRC values in Eq. (8), and then recalculate the DRC values when a better material is found by using full DFT for that new material, and proceed from that point by treating this as the new reference material. This is somewhat like doing the integral between Eqs. (3) and (4) above in a stepwise manner, keeping $X_{i}$ constant for small changes, but using new $X_{i}$ values in each such step. Thus, one can imagine tiling out descriptor space with small squares in this way, using the same DRCs within each tile, until one finds the optimum material. 

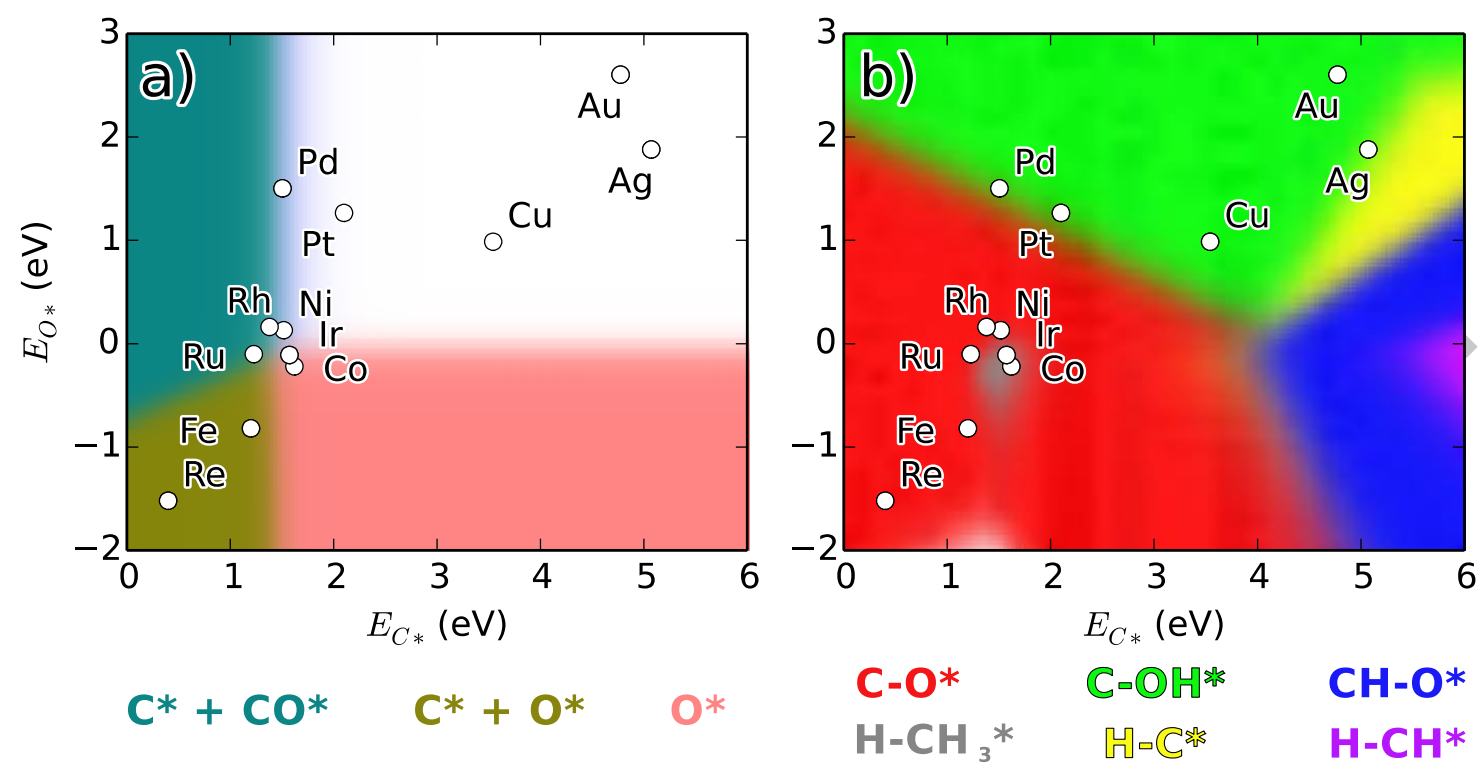

Figure 5: (a) The coverages (or DRCs) of adsorbed species and (b) the DRCs of transition states for the NB rate volcano in Fig 1a. The colors in the legend labels on the bottom left represent values of absolute magnitude 1.0, with lighter coloring being smaller coverages down to 0.0 (white). The coverages are equal to the absolute magnitude of the degree of rate control for adsorbed species, and the color map for coverages is created by letting the red, green, and blue channels be weighted by the coverage of $\mathrm{O}^{*}, \mathrm{C}^{*}$, and $\mathrm{CO}^{*}$ respectively. The color map for transition states is created similarly, with the color corresponding to each transition-state given to the bottom right corresponding to that $\mathrm{DRC}=1.0$.

Similarly, one could imagine improving the accuracy of the NB method by using full DFT instead of linear scaling to calculate the energies for all species with large DRCs, but retaining much of the speed by using linear scaling for all other species.

The DRC method certainly would not identify a better catalyst if the reference material were already on the maximum of a true volcano plot like Fig. 1a. However, since the NB method is the only way to generate such a volcano plot now, and since it has the inaccuracies associated with the linear scaling assumptions, the DRC method could in principal find a better catalyst than its estimated maximum. 
Using the scaling relations in conjunction with the DRC method allows one to visualize the full four-descriptor DRC approach in two dimensions. That is, the scaling relations are used to estimate the energies of all intermediates, and all species with a non-zero degree of rate control are included in Eq. (8) to estimate the rate at all points in the $\left(E_{C^{*}}, E_{O^{*}}\right)$ plane. The results of this method, along with a comparison to the NB rate volcano in Fig. 1a, are shown in Fig. 6. The addition of more species to the DRC method increases the accuracy very close to Rh, and in regions where the $\mathrm{C}^{*}$ coverage is high; however, the accuracy decreases overall, due to the fact that the $\mathrm{C}^{*}$ coverage becomes low for noble metals and thus the assumption of constant degree of rate control fails for these distant systems (see Fig 5a). This explains the unexpected decrease in accuracy with the addition of more species seen in Fig. 2a and Fig. 3.
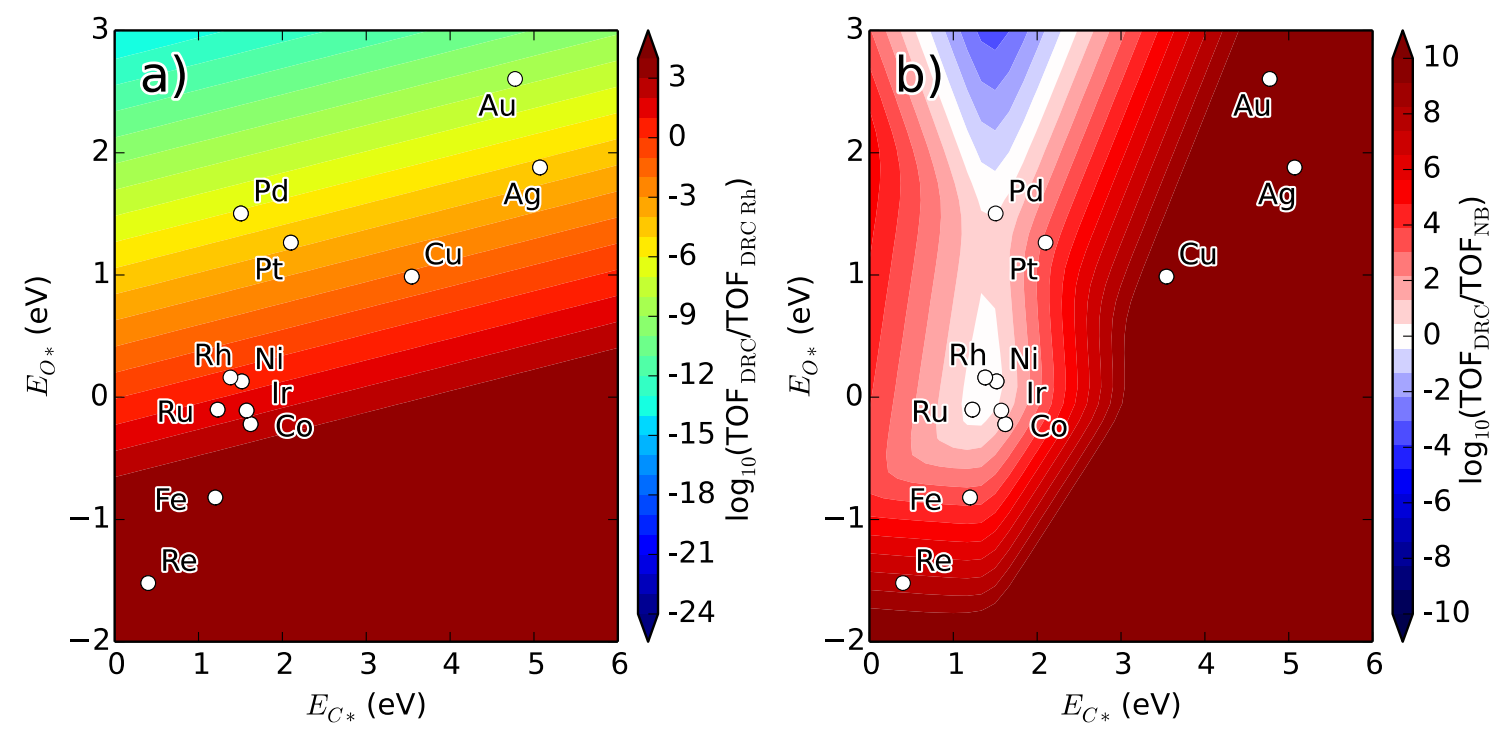

Figure 6: (a) Rates on the (211) facets of different metals computed with the DRC method using all four species with non-negligible $\mathrm{DRCs}$ on $\mathrm{Rh}(211)\left(\mathrm{C}-\mathrm{O} *, \mathrm{CO} *, \mathrm{C}^{*}\right.$ and $\mathrm{H}_{-} \mathrm{CH}_{3} *$ ), but now modified from Fig. 3 by using linear scaling relations to estimate the energies of these four descriptors rather than using DFT energies directly. Conditions are the same as Fig 1a.

(b) The ratio of these rates to rates from the NB method of Fig. 1a.

Note that the degrees of rate control for the reference material can be estimated experimentally as well. For example, the degrees of rate control for adsorbed intermediates are proportional to their coverages[13], which can be measured. Additionally when there is a single 
rate determining step, as frequently has been determined by experiments, the DRC for its transition state is 1.0. Thus, the new approach presented here also allows one to computationally search for better catalyst materials without even developing a full microkinetic model for the reference material, or even knowing the complete mechanism. This is because Eq. (8) allows one to estimate the rates for new materials using only the degrees of rate control and the energies of the most rate controlling species. Indeed, people have tried to improve catalysts for decades by changing the material to stabilize the rate-determining transition state or destabilize the dominant species that poisons sites. They have also looked for new materials that do this by using DFT. Thus, for the simple case where there is only a single rate-determining step and/or a single dominant adsorbate that poisons sites, this DRC method formalizes something that has already been done. But for more complex situations where there are more than two such species whose energies are important, it provides a fast and quantitative way to identify materials that improve the rate or selectivity.

\section{Computational Time}

One potentially big advantage of this new approach is its computational efficiency when screening materials for catalytic activity. It is far simpler to implement since, after the degree of rate control has been computed for the reference material, it is no longer necessary to use a kinetic model or solve complex differential equations for the other materials. Furthermore, it does not require the lengthy process of developing the many linear scaling relations needed for the NB approach. In order to more explicitly investigate these advantages we present a semiquantitative analysis of the computational time necessary for both methods.

The time $(\mathrm{t})$ required beyond the reference material for the NB method can be estimated as:

$$
t=\left(M *\left(N_{I}+\alpha N_{T}\right)+2 * M_{S}\right) t_{0} .
$$

where $t_{0}$ is the approximate time for a single adsorption energy calculation (by DFT, for example), $N_{I}$ is the number of adsorbed intermediates in the full mechanism, $N_{T}$ is the number of transition-states, $\alpha$ is the average ratio of computational time needed for one transition state energy relative to that for one adsorbate, $M$ is the number of additional material surfaces used to develop the linear scaling relationships and $M_{S}$ is the number of new material surfaces to be screened for rates. 
The approximate time needed for catalyst screening using the DRC method can then be estimated as:

$$
t=\left(M_{S} *\left(N_{I, K}+\alpha N_{T, K}\right)\right) t_{0}
$$

where $N_{I, K}$ and $N_{T, K}$ represent the number of key adsorbed intermediates and key transition-states with large DRCs whose energies are used when applying Eq. (8) (typically 1 or 2 of each).

Figure 7 compares the computational time needed for these two approaches for typical values of the parameters in Eqs. (9)-(10). The new method of this paper (blue curve) leads to major speedup when only a moderate number of materials are screened $(<50)$, and remains faster even up to 100 materials. However, it is actually slower for a large number of materials $(>150)$ due to the additional computational cost of calculating these key transition-states for so many materials. One can also use linear scaling relationships to estimate the energies of the key transition-states needed in this new method. These relations are less accurate, as discussed above; however, using them leads to a much-reduced computational time requirement for the DRC method, given by:

$$
t=\left(M *(2+\alpha)+M_{S} *\left(N_{A}^{\prime}+2 N_{T}^{\prime}\right)\right) t_{0} .
$$

Figure 5 (red curve) demonstrates that when using linear scaling for transition states only (i.e., using linear Brønsted relations only), the DRC approach remains faster than the NB approach up to a much larger numbers of screened materials $(\sim 1000)$. It is worth pointing out explicitly that the factor having the greatest impact on where these lines intersect is the value of $\alpha$, and that as $\alpha$ gets smaller the intersection points will push to higher numbers of screened materials, and vice versa.

The improvements in screening time and accuracy, and the simplicity of the DRC method make it an excellent complement to the traditional approach of catalyst screening with microkinetic models. However, it is not as robust, since it only works for relatively similar materials (descriptor differences $<0.5 \mathrm{eV}$, as quantified in Fig. 3). 


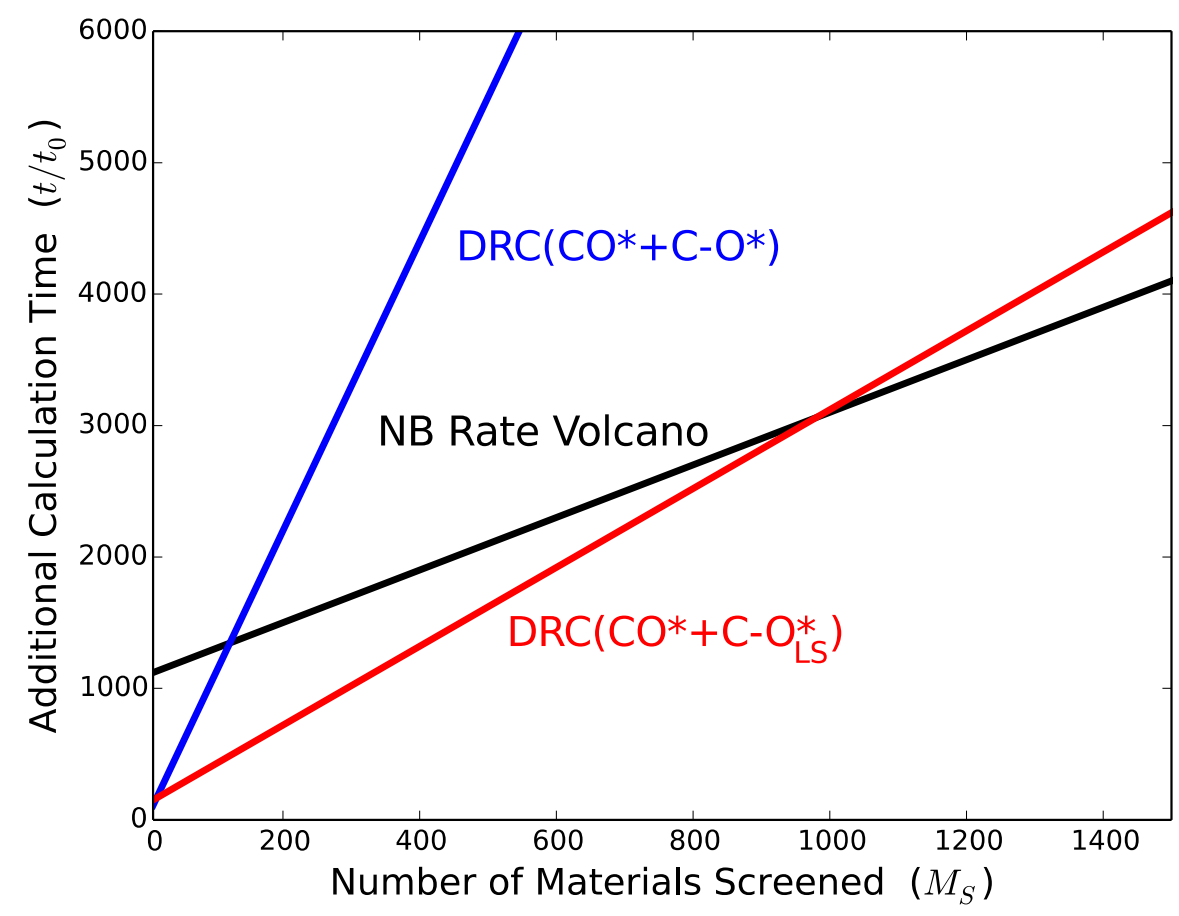

Figure 7. Comparison of estimated computer time needed for catalyst screening as a function of the number of surfaces to be screened. The black line shows the time for the NB approach. The blue line shows the integrated DRC method with transition-states computed using DFT, while the red line assumes that transition-state energies are estimated from linear scaling relationships. For this plot, reasonable values of $\mathrm{N}_{\mathrm{I}}=\mathrm{N}_{\mathrm{T}}=\mathrm{M}=\alpha=10$ and $\mathrm{N}_{\mathrm{I}, \mathrm{K}}=\mathrm{N}_{\mathrm{T}, \mathrm{K}}=1$ were used. These numbers will differ from system to system, and in particular $\alpha$ will vary considerably depending on the transition-state search algorithm used. However, the qualitative behavior shown here will remain.

\section{Conclusions}

An intuitive new method for computational catalyst screening is presented which is based on the concept of degree of rate control. The accuracy and computational efficiency of the method is compared, at the same level of DFT, to the Nørskov-Bligaard (NB) method which uses linear scaling relationships to estimate the energies of all adsorbed intermediates and transition states. By focusing only on the species with large degrees of rate control, the number of species whose energies need to be calculated can be reduced significantly. The method is used to reanalyze the data of $\mathrm{Xu}$ et al. who studied methane steam reforming using the NB approach. The results of the new method agree very well with NB approach for metals that are similar to the 
reference metal $(\mathrm{Rh})$, and are even more accurate when the metals are $<0.5 \mathrm{eV}$ from the reference catalyst in the NB "descriptor space" (i.e., on a plot where the axes are the bond energies to $\mathrm{C}$ and $\mathrm{O}$ adatoms), but much less accurate when the materials are very different $(>1.5$ $\mathrm{eV}$ from the reference catalyst). The method is computationally faster than the NB method when fewer than 100 materials are screened, and mathematically simpler. It thus shows great potential as a tool to aid in new catalyst discovery. Because it is less robust than the NB approach with respect to analyzing very different materials, it will not replace the NB method but instead be an important complementary approach.

\section{Acknowledgements}

The authors thank Lars Grabow and Suljo Linic for insightful discussions. The authors acknowledge support for this work by the US Department of Energy Office of Basic Energy Sciences (DOE-OBES) Materials Genome Initiative (MGI) by a subcontract through the Stanford University (Principal Investigator: Jens K. Norskov), and by the DOE EFRC Inorganometallic Catalyst Design Center (grant number DE-SC0012702, Principal Investigator Laura Gagliardi at the University of Minnesota). AJM is grateful for support by the Department of Defense (DoD) through the National Defense Science \& Engineering Graduate Fellowship (NDSEG) Program. A.J.M. and F.S. gratefully acknowledge support from the US Department of Energy, Office of Sciences, Office of Basic Energy Sciences to the SUNCAT Center for Interface Science and Catalysis. 


\section{References}

[1] G. Ertl, Reactions at Solid Surfaces, John Wiley \& Sons, Inc., Hoboken, NJ, 2009.

[2] J.M. Thomas, W.J. Thomas, Principles and practice of heterogeneous catalysis, Weinheim, New York, 2008.

[3] J.K. Norskov, T. Bligaard, J. Kleis, Science 324 (2009) 1655.

[4] H.W. Turner, A.F. Volpe, W.H. Weinberg, Surface Science 603 (2009) 1763.

[5] J.K. Norskov, F. Abild-Pedersen, F. Studt, T. Bligaard, Proceedings of the National Academy of Sciences of the United States of America 108 (2011) 937.

[6] F. Studt, F. Abild-Pedersen, T. Bligaard, R.Z. Sorensen, C.H. Christensen, J.K. Norskov, Science 320 (2008) 1320.

[7] J. Greeley, M. Mavrikakis, Nature Materials 3 (2004) 810.

[8] J. Greeley, I.E.L. Stephens, A.S. Bondarenko, T.P. Johansson, H.A. Hansen, T.F.

Jaramillo, J. Rossmeisl, I. Chorkendorff, J.K. Norskov, Nature Chemistry 1 (2009) 552.

[9] M.P. Andersson, T. Bligaard, A. Kustov, K.E. Larsen, J. Greeley, T. Johannessen, C.H. Christensen, J.K. Norskov, Journal of Catalysis 239 (2006) 501.

[10] J. Greeley, T.F. Jaramillo, J. Bonde, I.B. Chorkendorff, J.K. Norskov, Nature Materials 5 (2006) 909.

[11] S. Alayoglu, A.U. Nilekar, M. Mavrikakis, B. Eichhorn, Nature Materials 7 (2008) 333.

[12] F. Studt, I. Sharafutdinov, F. Abild-Pedersen, C.F. Elkjaer, J.S. Hummelshoj, S. Dahl, I. Chorkendorff, J.K. Norskov, Nature Chemistry 6 (2014) 320.

[13] C. Stegelmann, A. Andreasen, C.T. Campbell, Journal of the American Chemical Society 131 (2009) 8077.

[14] F. Abild-Pedersen, J. Greeley, F. Studt, J. Rossmeisl, T.R. Munter, P.G. Moses, E. Skulason, T. Bligaard, J.K. Norskov, Physical Review Letters 99 (2007) 4.

[15] T. Bligaard, J.K. Norskov, S. Dahl, J. Matthiesen, C.H. Christensen, J. Sehested, Journal of Catalysis 224 (2004) 206.

[16] J.K. Norskov, T. Bligaard, A. Logadottir, S. Bahn, L.B. Hansen, M. Bollinger, H. Bengaard, B. Hammer, Z. Sljivancanin, M. Mavrikakis, Y. Xu, S. Dahl, C.J.H. Jacobsen, Journal of Catalysis 209 (2002) 275.

[17] J.K. Norskov, T. Bligaard, J. Rossmeisl, C.H. Christensen, Nature Chemistry 1 (2009) 37.

[18] P. Ferrin, A.U. Nilekar, J. Greeley, M. Mavrikakis, J. Rossmeisl, Surface Science 602 (2008) 3424.

[19] A.C. Lausche, J.S. Hummelshoj, F. Abild-Pedersen, F. Studt, J.K. Norskov, Journal of Catalysis 291 (2012) 133.

[20] A. Michaelides, Z.P. Liu, C.J. Zhang, A. Alavi, D.A. King, P. Hu, Journal of the American Chemical Society 125 (2003) 3704.

[21] C.T. Campbell, Journal of Catalysis 204 (2001) 520.

[22] C. Stegelmann, N.C. Schiodt, C.T. Campbell, P. Stoltze, Journal of Catalysis 221 (2004)

630.

[23] S. Kozuch, S. Shaik, Journal of the American Chemical Society 128 (2006) 3355.

[24] H. Meskine, S. Matera, M. Scheffler, K. Reuter, H. Metiu, Surface Science 603 (2009) 1724 . 
[25] M.A. Sanchez-Castillo, N. Agarwal, C. Miller, R.D. Cortright, R.J. Madon, J.A. Dumesic, Journal of Catalysis 205 (2002) 67.

[26] S. Linic, M.A. Barteau, Journal of Catalysis 214 (2003) 200.

[27] C.T. Campbell, J.R.V. Sellers, Journal of the American Chemical Society 134 (2012) 18109.

[28] C.T. Campbell, L. Arnadottir, J.R.V. Sellers, Zeitschrift Fur Physikalische ChemieInternational Journal of Research in Physical Chemistry \& Chemical Physics 227 (2013) 1435.

[29] J.K. Norskov, T. Bligaard, Angewandte Chemie-International Edition 52 (2013) 776.

[30] R.W. Howarth, A. Ingraffea, T. Engelder, Nature 477 (2011) 271.

[31] J.D. Holladay, J. Hu, D.L. King, Y. Wang, Catalysis Today 139 (2009) 244.

[32] J.N. Armor, Applied Catalysis a-General 176 (1999) 159.

[33] D.A. Hickman, L.D. Schmidt, Science 259 (1993) 343.

[34] G. Jones, J.G. Jakobsen, S.S. Shim, J. Kleis, M.P. Andersson, J. Rossmeisl, F. AbildPedersen, T. Bligaard, S. Helveg, B. Hinnemann, J.R. Rostrup-Nielsen, I. Chorkendorff, J. Sehested, J.K. Norskov, Journal of Catalysis 259 (2008) 147.

[35] Y. Xu, A.C. Lausche, S. Wang, T.S. Khan, F. Abild-Pedersen, F. Studt, J.K. Norskov, T. Bligaard, New Journal of Physics 15 (2013) 125021.

[36] A.J. Medford, J. Sehested, J. Rossmeisl, I. Chorkendorff, F. Studt, J.K. Norskov, P.G. Moses, Journal of Catalysis 309 (2014) 397.

[37] A.J. Medford, C. Shi, M.J. Hoffmann, A.C. Lausche, S.R. Fitzgibbon, T. Bligaard, N. J.K., Catalysis Letters, 2015.(DOI 10.1007/s10562-015-1495-6).

[38] M.W. Chase, T. National Institute of Standards and, NIST-JANAF thermochemical tables, American Chemical Society ; American Institute of Physics for the National Institute of Standards and Technology, [Washington, D.C.]; Woodbury, N.Y., 1998.

[39] C.H. Shomate, Journal of Physical Chemistry 58 (1954) 368.

[40] A.J. Medford, A.C. Lausche, F. Abild-Pedersen, B. Temel, N.C. Schjodt, J.K. Norskov, F. Studt, Topics in Catalysis 57 (2014) 135.

[41] A.C. Lausche, A.J. Medford, T.S. Khan, Y. Xu, T. Bligaard, F. Abild-Pedersen, J.K. Norskov, F. Studt, Journal of Catalysis 307 (2013) 275.

[42] J.A. Herron, J. Jiao, K. Hahn, G.W. Peng, R.R. Adzic, M. Mavrikakis, Electrocatalysis 3 (2012) 192.

[43] R.G. Zhang, L.Z. Song, Y.H. Wang, Applied Surface Science 258 (2012) 7154.

[44] J.S. Yoo, F. Abild-Pedersen, J.K. Norskov, F. Studt, Acs Catalysis 4 (2014) 1226.

[45] F. Studt, F. Abild-Pedersen, Q.X. Wu, A.D. Jensen, B. Temel, J.D. Grunwaldt, J.K. Norskov, Journal of Catalysis 293 (2012) 51.

[46] C.J.H. Jacobsen, S. Dahl, B.S. Clausen, S. Bahn, A. Logadottir, J.K. Norskov, Journal of the American Chemical Society 123 (2001) 8404.

[47] T.F. Jaramillo, K.P. Jorgensen, J. Bonde, J.H. Nielsen, S. Horch, I. Chorkendorff, Science 317 (2007) 100.

[48] P.G. Moses, L.C. Grabow, E.M. Fernandez, B. Hinnemann, H. Topsoe, K.G. Knudsen, J.K. Norskov, Catalysis Letters 144 (2014) 1425. 


\section{Graphical Abstract:}

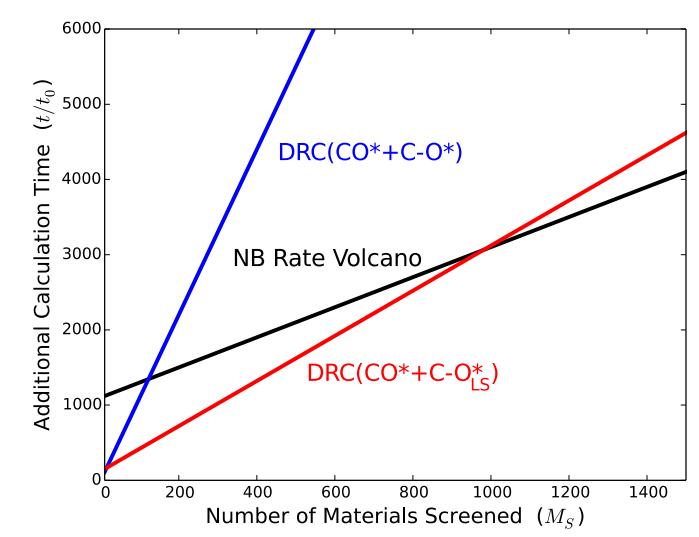

\section{Highlights:}

- Fast and intuitive new method for computational catalyst screening introduced.

- Uses the energies of the few species with the highest degrees of rate control (DRCs) for a reference catalyst as descriptors to estimate rates on related materials.

- Rate predictions for 12 transition metals for methane steam reforming compared to the standard Nørskov-Bligaard (NB) method.

- More accurate than NB method when the metals are similar to the reference metal $(<0.5 \mathrm{eV}$ different in descriptor space, i.e., on a plot where the axes are the bond energies to $\mathrm{C}$ and $\mathrm{O}$ adatoms).

- Much less accurate than the NB method when the materials are very different $(>1.5 \mathrm{eV})$.

- Computationally faster than NB method when screening <100 new materials. 